\title{
Microdiamonds from the Yubileinaya kimberlite pipe, Yakutia: morphology, physical properties, and mineral inclusions
}

\author{
Logvinova, A.M., Fedorova, E.N., and Sobolev, N.V.
}

Institute of Mineralogy and Petrography, Siberian Branch of Russian Academy of Sciences, Novosibirsk, 630090, Russia

Introduction. The origin of microdiamonds (crystals $<1 \mathrm{~mm}$ ) is widely debated by various researchers. Microdiamonds intrigue the researchers by their role in the genesis of macrodiamonds. The most reliable evidence of the conditions of diamond growth comes from mineral inclusions trapped in the process of their growth and from study of structural admixture centers. We have examined 2040 unbroken crystals, amounting about $50 \%$ of the whole collection of microdiamonds from the Yubileinaya kimberlite pipe, Yakutia. The specimens having lost more than one third of crystal were considered fragments and excluded from examination.

The Yubileinaya pipe is situated in the central part of the Daldyn-Alakit kimberlite field of the Yakutian diamondiferous province. The pipe age determined by the zircon-based U-Pb method is 358.1 Ma (Davis et al., 1980).

Methods. Specific features of the outer morphology and microrelief of microcrystals were studied by means of the JSM-35 scanning electron microscope. In addition, some morphological features of microdiamonds and inclusions in them were examined by the Zeiss Axiolab polarization microscope. Some physical properties of microdiamonds were characterized on the basis of absorption spectra in the IR-, UV-, and visible domains. The IR-spectra were obtained by the Perkin-Elmer 325 spectrophotometer with the help of a special accessory for microsamples. The UV and visible spectra are recorded on the Specord M40 spectrophotometer. The chemical composition of inclusions is determined with the Camebax-micro, electron probe.

Morphology. Microdiamonds are various in shape. Crystals of octahedral, dodecahedral, and cubic habits, rounded crystals, random and regular intergrowths, as well as microaggregate formations. Regular natural crystals of diamond are relatively rare. As usual, these are octahedra. In the studied pipe they amount $18 \%$. However, subjected to close examination, even apparently perfect plane-face and sharp-edge octahedra appear to be either slightly flattened along one of the axes $\mathrm{L}_{3}$ or slightly extended in the direction of one of the axes $\mathrm{L}_{2}$. Octahedron faces are often of polycentric structure. The surfaces of octahedron faces of some crystals were completely patterned with triangular pits oriented to follow the outlines of $\{111\}$ faces. Dodecahedroids amount $31 \%$. Among the sculptures on the $\{110\}$ surfaces of microdiamonds, the most abundant are drop-like hills, splintery and, more rarely, sheaf-like striation where the axes $\mathrm{L}_{3}$ come to the surface. Crystals of this kind are characterized by bands of plastic deformation. Cuboids are the most rare group of crystals, amounting as little as $1 \%$. As a rule, their faces are convex and sculptured with coarse striation.

The most microdiamonds from the Yubileinaya Pipe are polycrystalline aggregates (40\% polycrystalline aggregates and $10 \%$ twins). This group includes spinel-twinned and irregular intergrowths. The former consist of two or more individuals with parallel elements of symmetry of crystals of octahedron habit. The irregular intergrowths are composed of a great number of varioussized crystals. Abundant mineral inclusions are observed in these aggregates.

Infrared results. The predominant nitrogen centers in the microdiamonds from the Yubileinaya Pipe are A and B1. There are no significant amounts of C-centers. Six per cent of octahedral crystals and $25 \%$ of dodecahedroids contain a hydrogen admixture (lines 3107 and $1405 \mathrm{~cm}^{-1}$ in $\mathbb{R}$ spectrum). The $\mathrm{N}_{3}$ and $\mathrm{N}_{9}$ band systems are noticed in the absorption spectra of crystals in the UV and visible spectra. 
According to the nitrogen content and aggregation, the studied microdiamonds are subdivided into several groups within one pipe. The nitrogen-free crystals (type II) amount $53 \%$ among the Yubileinaya octahedral microdiamonds (for comparison, in the Udachnaya and Sytykanskaya pipes their amount is not more than $4 \%$ ) (Zedgenizov et al., 1998). According to data of cathode luminescence, these crystals are of block structure. For $\mathrm{N}$-containing octahedra the predominant center is $\mathrm{Bl}$, while dodecahedroids are represented by crystals $\mathrm{IaA}-\mathrm{IaB}$. Microdiamonds of type II are quite rare among dodecahedroids. Cuboids contain relatively more nitrogen than the rest of the crystals. The overwhelming majority of cuboids contain nitrogen only in the form IaA. In the specimen $\mathrm{Yu}-1$ which is a cuboid enriched in nitrogen (3300 ppm) a high content of hydrogen is observed (absorption bands 3107 and $1405 \mathrm{~cm}^{-1}$ ).

Thus, nitrogen content and degree of its aggregation in microdiamonds from the Yubileinaya pipe are different. Probably this is evidence of different conditions of growth of microdiamonds even within one pipe.

Mineral inclusions. Olivine is the commonest inclusion and is documented in more than $85 \%$ of all the microdiamonds with inclusions under study. In five cases more than one olivine inclusion are examined within one diamond. With rare exception ( 2 cases) their compositions do not differ. The forsterite content varies from 86,6 to $93.9 \%$. In peculiar features of composition, the olivine inclusions from microdiamonds are generally close to the olivines from the macrodiamonds from Yakutian kimberlites (Fig.) (Sobolev et al., 1997; Meyer, 1987; Zedgenizov et al, 1998). However, $19 \%$ of the Yubileinaya microdiamonds contain more ferruginous olivines (Fo $=86,6-88,7 \%$ ).

In addition to olivines, there are inclusions of garnet, chromite, ilmenite, and a great deal of sulfides. Also, moissanite was found in one diamond.

Garnets belong to the U-type paragenesis (10.3\% $\mathrm{Cr}_{2} \mathrm{O}_{3}, 3.23 \% \mathrm{CaO}$ ). Chromites are $\mathrm{Cr}$-rich varieties (64.2 to $65 \mathrm{wt} . \% \mathrm{Cr}_{2} \mathrm{O}_{3}$ ). Ilmenites contain no $\mathrm{Mg}$ admixture, and their varieties of this kind have been
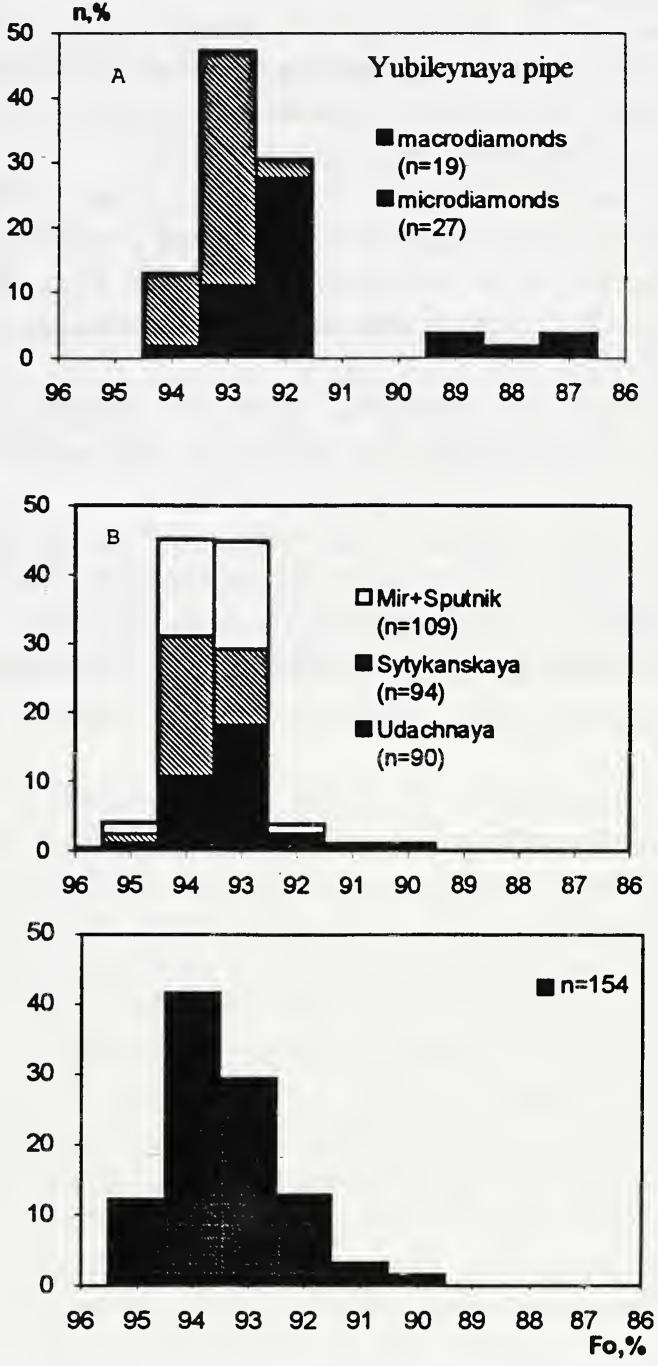

Fig. Forsterite content in olivines included in: macro- and microdiamonds from Yubileynaya (A); macrodiamonds from Mir,Sputnik, Sytykanskaya and Udachnaya pipe, Yakutia (B); macrodiamonds from various deposits of Asia, America, and Africa (Meyer, 1987) (C) .n - number of specimens. described elsewhere (Meyer, 1987).

In five cases silicate inclusions are observed; compositionally, they differ from the known silicate inclusions in the diamond. They are characterized by an elevated content of $\mathrm{K}_{2} \mathrm{O}$ (1.36-3.6 wt.\%). Inclusions of this composition were earlier described in cubic crystals and coated diamond (variety IV according to Orlov), where they are defined as inclusions of K-rich silicate melt (Schrauder, Navon, 1992). The presence of these inclusions in microdiamond is, probably, evidence of a greater, 
as compared with macrodiamonds, diversity of paragenetic associations and a more significant role of K-rich mineral-forming medium.

\section{References}

Davis, G.L., Sobolev, N.V., Kharkiv, A.D.,1980, New data on the age of Yakutian kimberlites obtained by the U-Pb method on zircons (in Russian): Dokl. Akad. Nauk SSSR, v.254, pp.175-179.

Meyer, H.O.A., 1987, Inclusions in diamonds. In P. H. Nixon (ed).Mantle Xenoliths, John Wiley and Sons, Chichester, England, pp.501-522.

Schrauder, M., Navon, O., 1992, Hydrous- and carbonatitic mantle fluids in fibrous diamonds from Jwaneng, Botswana: Geochemica and Cosmochemica Acta, v.52, N2, pp. 761-771.

Sobolev, N.V., Kaminsky, F.V, Griffin W.L., Yefimova, E.S., Win, T.T., Ryan, C.G., Botkunov, A.I., 1997, Mineral inclusions in diamonds from the Sputnik kimberlite pipe, Yakutia: Lithos, 39, pp. 135-157.

Trautman, R., Griffin,B., Taylor, W., Spetsius, Z., Smith, C., and Lee, D., 1997, A comparison of the microdiamonds from kimberlite and lamproite of Yakutia and Australia: Rusian Geology and Geophysics., v.38, N 2, pp. 341-355.

Zedgenizov, D.A., Logvinova, A.M., Shatsky, V.S., Sobolev, N.V., 1998, Inclusions in microdiamonds from some kimberlite pipes, Yakutia: Dokl. Acad. Nauk, v.359, No 1.

Zedgenizov, D.A., Fedorova, E.N., Shatsky, V.S., 1998, Microdiamonds from kimberlites of Udachnaya pipe: Russian Geology and Geophysics (in press). 